\title{
Structure of Dense Polymer Systems Confined to a Slit
}

\author{
A. SIKORSKI* \\ Department of Chemistry, University of Warsaw \\ Pasteura 1, 02-093 Warsaw, Poland \\ (Received 23 November, 2005)
}

\begin{abstract}
The linear polymer chains were approximated as series of identical segments on a simple cubic lattice. The excluded volume was included into the model with no attractive interactions which corresponded to the good solvent conditions. The polymer chains were put into a slit formed by a pair of parallel surfaces. These walls were impenetrable for polymer segments and no other interactions between walls and chains were assumed. The models chains were studied by the means of the Monte Carlo method. The sampling algorithm was Metropolis-type and employing micromodifications of chain's conformation to sample efficiently the conformational space. The influence of the chain length, density of the polymer system, and the distance between the surfaces on the shape of macromolecules was studied. It was found that the decrease in the size of the slit and the decrease in the polymer density led to the formation of more spherical macromolecules. This is partially caused by the interpenetration of polymer chains.
\end{abstract}

PACS numbers: 02.50.Ng, 05.10.Ln, 61.25.Hq

\section{Introduction}

The properties of confined polymer chains have been recently a subject of many experimental and theoretical works because of their practical importance, like size-exclusion chromatography, wetting, polymer adhesion, and lubrication [1]. Recently, the influence of the confinement and so-called molecular crowding (the presence of macromolecules of different kinds that occupy a considerable fraction of the total volume of a cell) on the biological activity and other properties of proteins focused the attention of theoretical studies [2]. Confined dense polymer systems (melts) were also studied by computer simulations and theoretical considerations [3]. Computer simulations of lattice and off-lattice models concerned mainly the influences of the polymer stiffness and the system density on chains'

*e-mail: sikorski@chem.uw.edu.pl 
size. The distributions of polymer segments in the slit and dynamic properties of macromolecules were also determined [4-6]. Recent studies of a cubic lattice model of polymer melts showed the universal behavior of the chain's size regardless of the system density and chain length, and determined their scaling behavior [7]. Dense polymer melts in attractive slits were also studied [8-11] and investigated in real experiments [12]. The introduction of the attractive slit led, at certain conditions, to the interesting swapping of fully adsorbed chains from one surface to another [13].

The present work is a further extension of the studies of simple models of dense polymer melts consisted of linear chains located in a slit formed by a pair of parallel and impenetrable surfaces. Because previously the question of polymer size was raised [7] now we focused our attention on the influence of the confinement and the density of the polymer system on the instantaneous shape of chains.

\section{The model and the method}

The model polymer chains were extremely simplified in order to make the calculations possible for larger and more dense systems. All the atomic details were suppressed and the macromolecules were approximated by chains built of united atoms called polymer segments. The locations and orientations of these segments were limited to vertices of a simple cubic lattice. The chains were flexible with no local stiffness introduced. The only potential of interactions was the excluded volume realized by the forbidding of the double occupancy of lattice sites (athermal system). The model chains were put between two impenetrable surfaces parallel to the plane $x y$. In directions of $x$ and $y$ axes the periodic boundary conditions were imposed [7, 14]. It was previously shown that such coarse models were sufficient for studying the properties of chain on the scale larger than a single segment [14]. Dense polymer melts consisting of $m$ chains each built of $N$ segments were the object of our studies. The density of the melt $\varphi$ was defined as the fraction of the slit's volume occupied by polymer segments [7].

The properties of model systems were studied by means of dynamic Monte Carlo method. In the simulation algorithm, the conformation of each polymer chain was randomly modified using the following set of micromodifications: 2-bond move, 3-bond move, 3-bond crankshaft move, 1-bond and 2-bond chain's ends moves. A set of motions consisting of one attempt of each move determines a time unit [14]. An attempt of a micromodification was accepted due to geometrical constraints and excluded volume caused by the chains as well as by the confining surfaces. The simulations were performed according to the following protocol. An initial conformation was built by the simultaneous growth and the equilibration of chains. Then, all chains underwent a series of local micromodifications $\left(10^{7}-10^{8}\right.$ time units). For each system under consideration $(N, M, d) 30-40$ independent Monte Carlo simulation runs were carried out starting from different initial conformations. 


\section{Results and discussion}

The chain length was varied between $N=100$ and $N=800$ segments, i.e. in the region where macromolecules were supposed to be entangled. The simulations were carried out for melt densities $\varphi$ between 0.1 and 0.5 . The size of the slit $d$ was changed between 50 and 3 lattice units in order to cover the regime from not distorted chain to flattened almost two-dimensional chain. The number of chains and the size of the Monte Carlo box $L$ along the directions parallel to the surfaces $(x$ and $y$ ) were changed in such a way as to maintain the constant concentration of the polymer segments in the slit. The edge of the box was varied between 43 and 136, while the number of chains was between 275 and 281 (for chains $N=100$ ) and between 137 and 140 (for chains $N=200$ ). Simulations of single confined linear chains of the same lengths at the same confinement were also carried out as a state of reference.

The first question concerns the influence of the slit's size on the exposure of chains on solvent and on the confining surfaces. The mean number of contacts between polymer segments and the surfaces can also be treated as a measure of the mobility of particular chain fragments. It was shown previously that the reduced number of contacts exhibits a universal behavior [15]. For our purposes we do not have to use the reduced quantities with the exception of segment numbering: in order to make the comparison between chains of different lengths we used the reduced segment numbering $i / N$, where $i$ is a number of an $i$ th segment. Figure 1 presents the mean number of contacts between polymer segments and surfaces as a function of reduced segment number. One can observe that numbers of contacts are almost constant (with some fluctuations) for the segments located in the middle of chains. The numbers of contacts for very ends of chains are considerably larger which is apparentlycaused by their higher mobility. The increase in the size of the

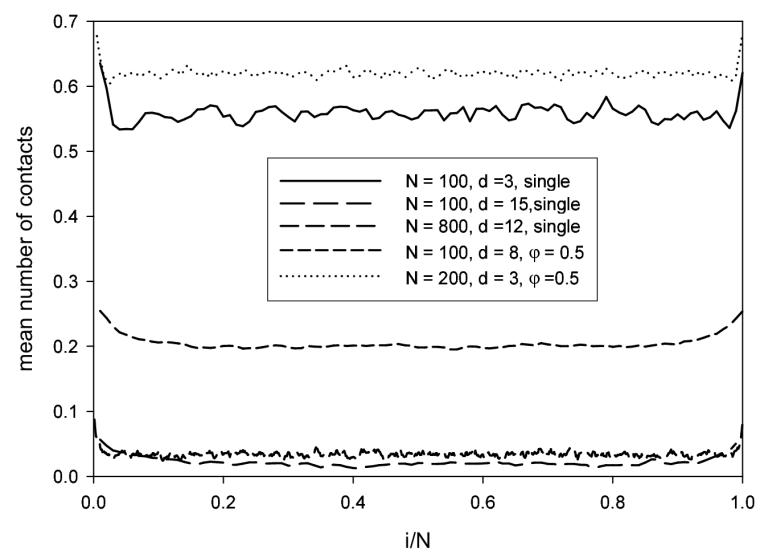

Fig. 1. The mean number of polymer segment-surface contacts versus the reduced segment number. The chain lengths, the size of the slit, and the density are given in the inset. 
slit makes these more mobile fragments longer and diminishes considerably the number of contacts.

The next question deals with the number of contacts between segments of the same chain (intrachain contacts) for different conditions under consideration. The mean numbers of these contacts are showed in Fig. 2. The behavior of this parameter is similar to those of polymer-surface contacts, i.e. for the entire middle part of chains the number of contacts is constant although the number of contacts is considerably greater. Here the dominant factor is the size of the slit.

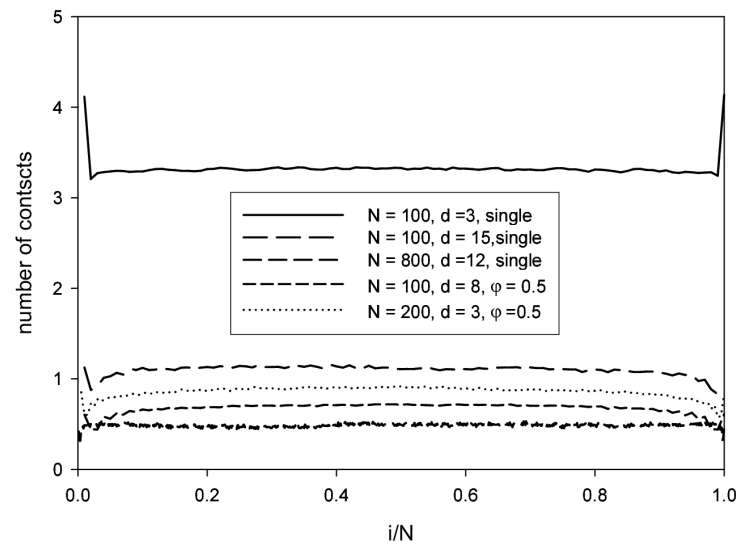

Fig. 2. The mean number of intrachain contacts versus the reduced segment number. The chain lengths, the size of the slit, and the density are given in the inset.

It is possible to extract much more data for the polymer chain from the radius of gyration tensor as one can determine the instantaneous shape of a macromolecule $[14,16]$. Let us consider the tensor of second kind

$$
S=\left|\begin{array}{ccc}
S_{x x} & S_{x y} & S_{x z} \\
S_{x y} & S_{y y} & S_{y z} \\
S_{x z} & S_{y z} & S_{z z}
\end{array}\right|,
$$

where its elements are defined as follows:

$$
S_{x x}=\sum_{i=1}^{N}\left(x_{x \mathrm{~m}}-x_{i}\right)\left(x_{\mathrm{cm}}-x_{i}\right), S_{x y}=\sum_{i=1}^{N}\left(x_{x \mathrm{~m}}-x_{i}\right)\left(y_{\mathrm{cm}}-y_{i}\right), \ldots
$$

and the subscript "cm" denotes center-of-mass. The diagonalization of the tensor $\boldsymbol{S}$ gives us eigenvalues $L_{1}^{2}, L_{2}^{2}, L_{3}^{2}$, which correspond to three main axes of the chain's equivalent ellipsoid. One can express the shape of the chain by giving the ratio of these axes. The equivalent ellipsoid of a polymer chain can also be determined experimentally by the small-angle X-ray scattering [17]. Monte Carlo simulations led to the following approximate ratio for the linear random flight chains (without the excluded volume) [18]: 


$$
L_{1}^{2}: L_{2}^{2}: L_{3}^{2}=8: 3: 1 .
$$

This result means that a polymer coil does not have the spherical shape but it is bean-like. The introduction of the excluded volume into the model leads to the greater elongation of the polymer coil which was confirmed by computer simulations [19]:

$$
L_{1}^{2}: L_{2}^{2}: L_{3}^{2}=14.8: 3.1: 1 \text {. }
$$

The operations on the above ratios are rather inconvenient. Therefore, usually one uses other parameters like shape factors and asphericity. Shape factors $s f_{i}$ are the normalized axes of the $i$ th ellipsoid:

$$
s f_{i}=\frac{L_{i}^{2}}{L_{1}^{2}+L_{2}^{2}+L_{3}^{2}} .
$$

Figure $3 \mathrm{a}-\mathrm{c}$ presents the shape factors for some polymer systems studied. One can observe that the longest axis, i.e. $s f_{1}$ parameter behaves in a different way as two remaining shape factors. The ratio of the shape parameters for larger slits is close to that predicted theoretically (Eq. (3b)). The $s f_{1}$ parameter has a
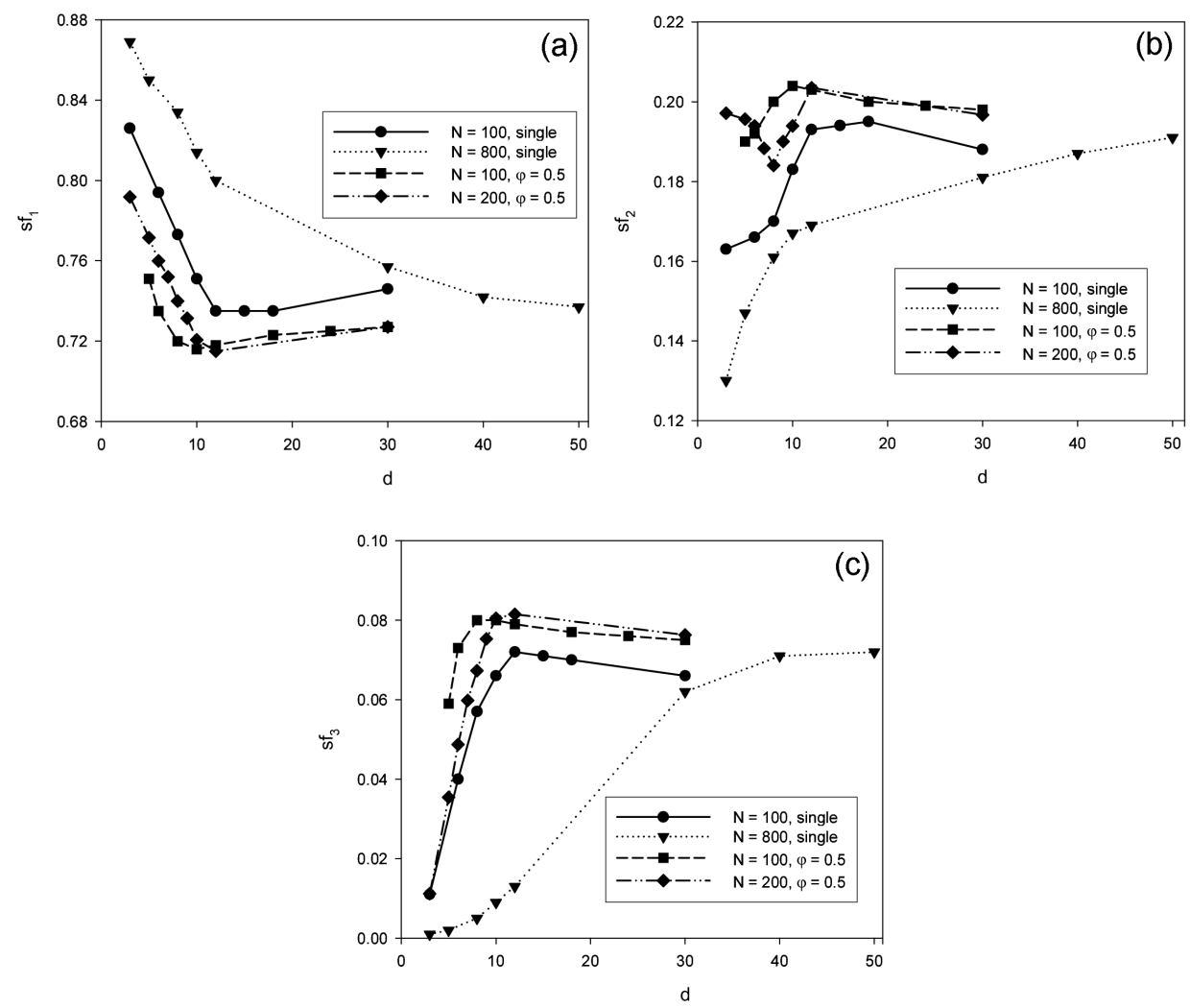

Fig. 3. The shape factors versus the distance between walls $d: s f_{1}$ (a), $s f_{2}$ (b), and $s f_{3}$ (c). The chain lengths and the density are given in the insets. 
shallow minimum but the location of this minimum is shifted towards the stronger confinement (smaller $d$ values) when compared to the minimum on $\left\langle S^{2}\right\rangle$ and $\left\langle R^{2}\right\rangle$ curves [7]. Contrary to this axis of the equivalent ellipsoid two remaining parameters $s f_{2}$ and $s f_{3}$ have a maximum at the same value of $d$. Therefore, the net result of the decreasing of the slit size is that polymer chains become rather two-dimensional with a high asymmetry. The location of the extrema on shape factors curve is shifted towards higher $d$ when the length of chain increases. The increase in the system density shifts these minima (maxima) in the opposite direction. The direction of the shift is associated with the size of the chain: it increases with the chain length and decreases with the system density [7].

In order to avoid using 3 parameters describing the shape of polymer chains the so-called asphericity factor was introduced by Rudnick and Gaspari [20]. The asphericity factor $\delta^{*}$ is usually defined as:

$$
\delta=\frac{\left\langle\left(L_{1}^{2}-L_{2}^{2}\right)^{2}+\left(L_{1}^{2}-L_{3}^{2}\right)^{2}+\left(L_{2}^{2}-L_{3}^{2}\right)^{2}\right\rangle}{2\left\langle\left(L_{1}^{2}+L_{2}^{2}+L_{3}^{2}\right)^{2}\right\rangle} .
$$

This parameter was designed in order to take the value 0 for the spherical shape and 1 for one-dimensional rod. The analytical values of the factor $\delta^{*}$ for a random-flight linear chain were given by Rudnick et al. [18] $\delta^{*}=0.526$ and by Aronovitz and Nelson [21] $\delta^{*}=0.534$. Computer simulations of linear chains without the excluded volume gave very similar results with $\delta^{*}=0.527$ while extrapolated to the infinite length of the chain. For finite chains the values of the parameter $\delta^{*}$ depended not only on their lengths but also on the model (a type of a lattice approximation used); the asphericity was located between 0.51 and 0.54 for chains consisting of more than $N=50$ segments [18].

Figure 4 presents the changes of the asphericity for single chains as well as for melts as a function of the size of the slit $d$. One can observe that the asphericity of chains decreases when going down with distance $d$ starting from smaller confinements and a minimum occurs, which could have been expected from the behavior of shape factors (see Fig. 3). The minimum on the curve for a single chain with $N=800$ segments appears for much larger $d$ values and, therefore, is not visible in this picture. For stronger confinements where chains are squeezed their asphericity is considerably larger. In general, the dependence of the asphericity factor on the width of the slit is similar to that of chain's size. The main difference is the localization of the minimum on the curve: for $\delta^{*}$ it is shifted towards a larger slit's size. This means that the influence of the distance between the surfaces begins earlier for the shape of chains than for the size of chains [7]. What is interesting is the fact that the influence of the segment density of the polymer system on the shape of chains is surprisingly weak and the length of chain is rather the main factor - longer the chains are, the asphericity of a polymer is larger. For polymer melts the increase in the chain length does not considerably change the asphericity for moderate confinements. For highly squeezed chains the difference in $\delta^{*}$ becomes more significant. 


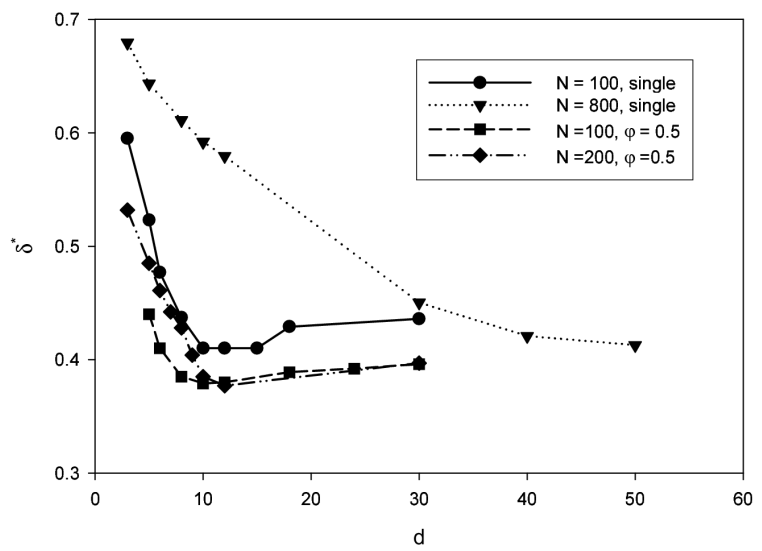

Fig. 4. The mean asphericity of the chain $\delta^{*}$ versus the distance between walls $d$. The chain lengths and the density are given in the inset.

It was shown that the reduced parameters enable one to construct a universal curve regardless of chain length, internal macromolecular architecture, and the density of the system $[7,14,15]$. One can also try to construct a master curve similar to that for the chain's size but concerning the instantaneous shape of a macromolecule. For this purpose we used the asphericity factors $\delta^{*}$ divided by the appropriate values for unconfined chains $\delta_{0}^{*}$. The reduced size of the slit was already defined as $[7,14]$ :

$$
d^{*}=\frac{d}{\left\langle S^{2}\right\rangle_{0}^{1 / 2}},
$$

where $\left\langle S^{2}\right\rangle_{0}$ is the mean squared radius of gyration for the unconfined chain (bulk conditions) of a given length and the system density. In Fig. 5 we present an attempt to create such a universal curve for the chain's instantaneous shape plotting the reduced asphericity $\delta^{*} / \delta_{0}^{*}$ versus the reduced size of the slit $d^{*}$. It appears that all curves presented in this figure are of similar shape but the deviations can be found for all polymer systems under consideration and for all confinements. These deviations can be even found for larger slits and for short chains. The asphericity of systems is almost constant above $d^{*}=1$ and has a minimum just below $d^{*}=1$ although the increase of the chain's asymmetry is not large. The further squeezing of polymers led to the rapid increase of the asphericity approaching the value of 1.4 .

The influence of the density of the polymer system in the slit on chains' shape is also an interesting problem. In Fig. 6 we present the asphericity factor $\delta^{*}$ as a function of the melt density $\varphi$. One can observe that the increase in the density leads to a significant decrease in chain's aspherictity factor. For longer chains the shape of macromolecules was more aspherical. One has to remember that the size of chain diminishes smoothly with the increase in the melt density and 


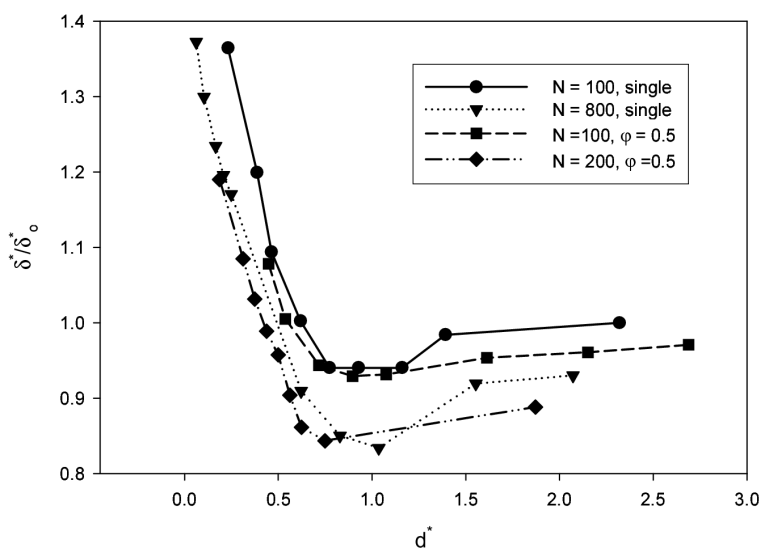

Fig. 5. The reduced mean asphericity $\delta^{*} / \delta_{0}^{*}$ of the chain versus the reduced distance between surfaces $d^{*}$. The chain lengths and the density are given in the inset.

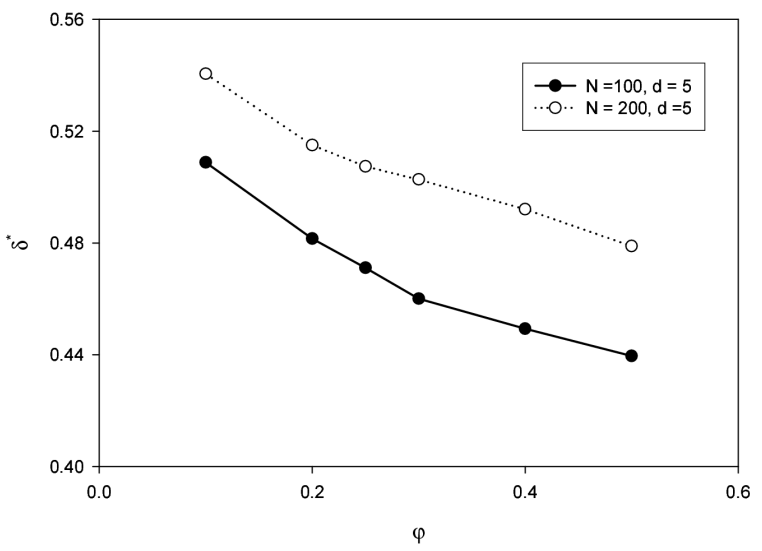

Fig. 6. The mean asphericity of the chain $\delta^{*}$ versus the density of the melt $\varphi$. The chain lengths and the size of the slit are given in the inset.

this decrease is the most rapid for highly squeezed chains [7]. Therefore, we can conclude that in the presence of macromolecular crowding the confined polymer chains have to be more spherical in spite of squeezing in the slit.

\section{Conclusions}

Properties of confined linear polymer chains were studied using simple and reduced lattice models. The behavior of single linear chains and of melts composed of such chains was determined. Polymer chains were constructed as a sequence of identical segments on a simple cubic lattice at good solvent conditions (no energetic contact was distinguished). The Metropolis-like Monte Carlo simulation algorithm used enabled us to study long chains up to 800 statistical segments and melts with the density of polymer segments of 0.5 . 
It was shown that the shape of confined chains differed from that for free (unconfined) chains. The moderately squeezed chains are less spherical than free chains regardless their length and the system density. Highly squeezed polymers are much more asymmetric than a chain at bulk conditions. The increase in the system density causes the chain interpenetration and makes macromolecules more spherical.

\section{References}

[1] I. Teraoka, Progr. Polym. Sci. 21, 89 (1996).

[2] G. Rivas, F. Ferrone, J. Hertzfeld, EMBO Rep. 5, 23 (2003).

[3] K. Binder, A. Milchev, J. Comput.-Aided Mater. 9, 33 (2002).

[4] G. ten Brinke, D. Ausserre, G. Hadziioannou, J. Chem. Phys. 89, 4374 (1988).

[5] T. Pakula, J. Chem. Phys. 95, 4685 (1991).

[6] A. Yethiraj, J. Chem. Phys. 101, 2489 (1994).

[7] A. Sikorski, Acta Phys. Pol. A 107, 443 (2005).

[8] D.V. Kuznetsov, A.C. Balazs, J. Chem. Phys. 113, 2479 (2000).

[9] Y. Yin, P. Sun, T. Chen, B. Li, Q. Jin, D. Ding, A.-C. Shi, Chem. Phys. Chem. 5, 540 (2004).

[10] I. Bitsanin, G. Hadziioannou, J. Chem. Phys. 92, 3827 (1990).

[11] T. Ayoagi, J. Takimoto, M. Doi, J. Chem. Phys. 115, 552 (2001).

[12] J.-M. Georges, A. Tonk, J.-L. Loubet, D. Mazuyer, E. Georges, F. Sidoroff, J. Phys II (France) 6, 57 (1996).

[13] P. Romiszowski, A. Sikorski, J. Chem. Phys. 123, 104905 (2005).

[14] A. Sikorski, Acta Phys. Pol. A 103, 339 (2003).

[15] A. Sikorski, P. Romiszowski, J. Chem. Phys. 120, 7206 (2004).

[16] K. Šolc, J. Chem. Phys. 55, 335 (1971).

[17] J.J. Müller, H. Schrauber, J. Appl. Cryst. 25, 181 (1992).

[18] A. Beldjenna, J. Rudnick, G. Gaspari, J. Phys. A, Math. Gen. 24, 2131 (1991).

[19] J. Mazur, C.M. Gutman, F.L. McCrackin, Macromolecules 6, 872 (1973).

[20] J. Rudnick, G. Gaspari, J. Phys. A, Math. Gen. 20, L191 (1986).

[21] J.A. Aronovitz, D.R. Nelson, J. Phys. (Les Ulis, Fr.) 47, 1445 (1986). 